\title{
The Post-Editing Workflow: Training Challenges for LSPs, Post-Editors and Academia
}

\author{
Viveta Gene ${ }^{[0000-0002-4374-8305]}$ \\ Ionian University, Corfu, Greece \\ viveta.gene@gmail.com
}

\begin{abstract}
Language technology is already largely adopted by most Language Service Providers (LSPs) and integrated into their traditional translation processes. In this context, there are many different approaches to applying Post-Editing (PE) of a machine translated text, involving different workflow processes and steps that can be more or less effective and favorable. In the present paper, we propose a 3-step Post-Editing Workflow (PEW). Drawing from industry insight, this paper aims to provide a basic framework for LSPs and Post-Editors on how to streamline Post-Editing workflows in order to improve quality, achieve higher profitability and better return on investment and standardize and facilitate internal processes in terms of management and linguist effort when it comes to PE services. We argue that a comprehensive PEW consists in three essential tasks: Pre-Editing, Post-Editing and Annotation/Machine Translation (MT) evaluation processes (Guerrero, 2018) supported by three essential roles: Pre-Editor, Post-Editor and Annotator (Gene, 2020). Furthermore, the present paper demonstrates the training challenges arising from this PEW, supported by empirical research results, as reflected in a digital survey among language industry professionals (Gene, 2020), which was conducted in the context of a Post-Editing Webinar. Its sample comprised 51 representatives of LSPs and 12 representatives of SLVs (Single Language Vendors) representatives.
\end{abstract}

Keywords: Post-editing workflow; training challenges; pre-editing; error annotation.

\section{Introduction}

The role of the post-editor was first mentioned almost thirty years ago. However, the skills, competences, tasks and processes related to this role need to be revised in light of the rising significance of MT (Rico, 2017) and the translation workflow needs to be human-centered to offer advantages for all stakeholders (Guerrero, 2018).

The use of Translation Memories (TMs) and MT in the localization workflow paved the way for the exploration of new methods of producing higher-volume translations at lower costs while maintaining quality. This resulted in new translation workflows including pre-editing and PE of raw output and the creation of new guidelines to support the work in this environment and the training of translators (Guerberof, 2017).

According to Guerberof (2017), even if the pre-editing and post-editing of raw output have been implemented in some organizations since the 1980s, it is only in the last ten years that Machine Translation Post-Editing (MTPE) has been introduced as a 
part of the standard translation workflow in most localization agencies worldwide (Lommel \& DePalma 2016a). As a result, the need of training in the ways that technology affects the standard translation workflow and the agents involved in the process becomes a priority.

\section{The Post-Editing Workflow}

In a digital survey among language industry professionals (Gene, 2020), which was conducted in the context of a Post-Editing Webinar, one of the questions was about the workflow in use by LSPs for Post-Editing. The results hereby presented reflect the answers of 51 LSPs (see Fig. 1):

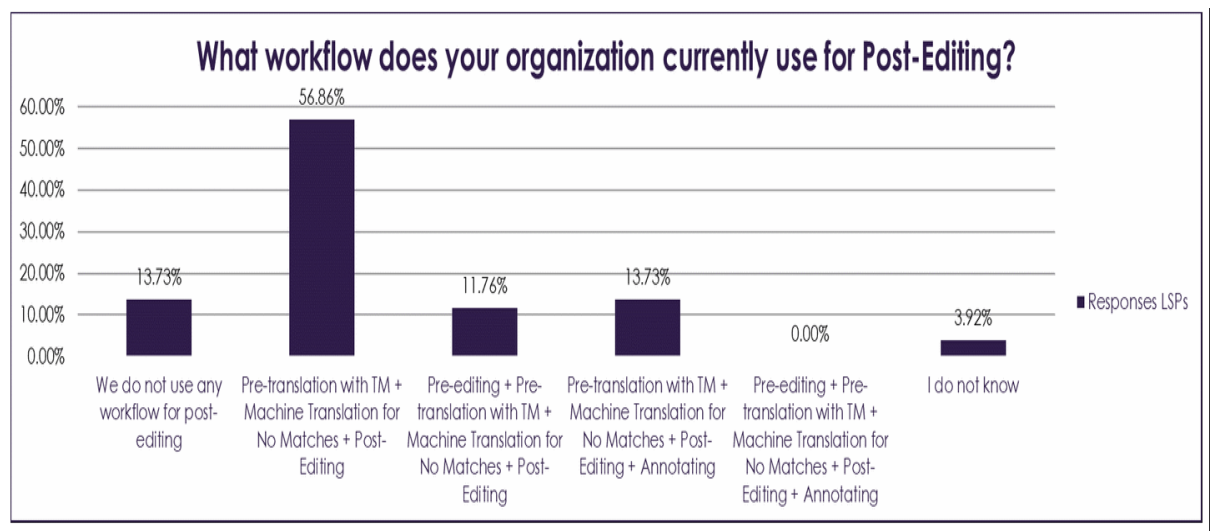

Fig. 1. GALA Survey, Question on the LSPs PEW

The responses to this question reveal the training challenges related to the PEW. They indicate that TM and MT technology are now well established in the workflows of LSPs, although LSPs and all other stakeholders (Post-Editors, Academia and Clients) either lack the training and budget needed or the training and adequate volumes/relevant nature of projects to be able to implement or make the most of a postediting workflow.

In this paper, we argue that a comprehensive PEW consists in three human-centered tasks (see Fig. 3): Pre-Editing, Post-Editing and Quality Checks/Annotation/MT evaluation processes (Gene, 2020). 


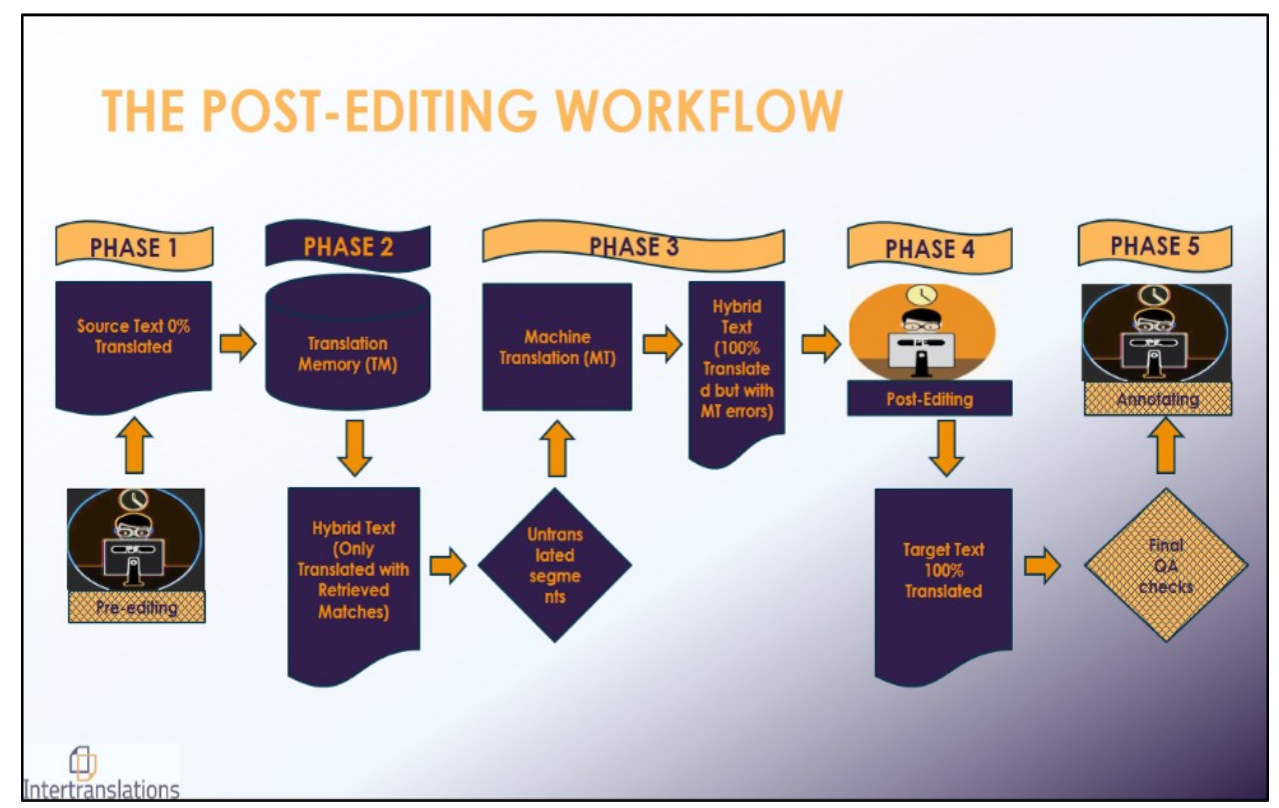

Fig. 1. The Post-Editing Workflow

It should be noted that not all translation projects are good candidates for a comprehensive 3-step PEW (O'Brien, 2003), as this depends on the language combination, specialization field, use-case scenario, nature, volume, budget, deadline and a collaborative post-editing protocol established between the LSP and the Client. This section provides the definitions of the three aforementioned human-centered tasks from an industrial as well as from an academic point of view as the starting point for research in the language industry.

\subsection{Pre-editing}

Pre-editing is performed using a set of terminological and stylistic guidelines or rules for the pre-processing of the source text before any translation automation in the scope of improving the raw output quality, therefore reducing the effort required on the part of the linguists to post-edit said output and increasing their productivity (Sanchez-Martinez, 2012). Besides, errors in the source text may prevent the translation system from finding the best matches for each segment (Guerrero, 2018).

According to O'Brien (2003), pre-editing is intended for very specific domains and companies. When combined with controlled language rules which aim to simplify and polish the source text, pre-editing ensures that the material to be processed is in the optimal condition to allow for the optimal quality of MT output production and minimal effort in the PE phase, providing lexical clarity and simplification of complex grammatical structures (Sanchez-Martinez, 2012). The goal is, in other words, to end up with a source that will be well "understood" by both the reader and the MT engine. 
In this aspect, we may consider "controlled language" as a way of expression that is more compatible with the MT engine's own "language".

For optimal results, these methods should be combined with other technology tools: Computer-Assisted Translation (CAT) tools should be integrated in the postediting workflow in a way that complements machine-generated results and ensures a superior level of accuracy and consistency in terms of terminology and domain-specific jargon.

After the aforementioned pre-editing steps are completed, the untranslated text is connected to an existing Translation Memory (TM) to leverage matches from previously translated content. This process results in a text partially translated only by TMretrieved segments. At this point, MT comes into play, with lowest scoring untranslated strings ( $0-50 \%$ matches) going through the engine to be translated.

The final product is an entirely translated text, which, however, contains MT errors that are going to be processed during the actual Post-Editing stage of the workflow. This hybrid text, combining matches retrieved from human-generated TM and (preferably customized) machine-translated output, is what should ideally reach the Post-Editor, in order to ensure minimal editing effort and superior linguistic quality.

\section{$2.2 \quad$ Post-editing}

Post-editing is to edit, modify and/or correct pre-translated text that has been processed by an MT system from a source language into (a) target language(s) (Allen, 2003). In the comprehensive PEW described here (see Fig. 2), Post-Editing follows the pre-editing step.

The focus here is on the linguist's tasks. These involve being able to quickly evaluate the text's elements (whether deriving from TM suggestions or MT output) and correct or eliminate errors, add any missing elements or remove redundant ones, while paying attention to terminology and to the text's overall fluency and style. This requires a rather clear understanding of the way the specific type of MT engine at hand functions and which kinds of errors it is most likely to generate. It is worth noting that in recent times, the focus of linguists and post-editing experts (and, on a broader level, the focus of Post-Editing training) is practically on NMT systems, which are all the more widely used and have replaced SMT or rule-based systems almost entirely (Blagodarna, 2018).

To summarize the points made so far, the key to this stage of the PEW is in the way MT errors are handled by the linguists. The goal is to adopt a balanced approach when selecting between available MT and TM suggestions and identifying the cases where translating from scratch would be required. Every option should be weighed against not only the quality of the final product but also the amount of effort required to process or generate it in order to increase productivity and to accelerate the translation process (Guerberof, 2018). 


\subsection{Quality Checks - MT Evaluation - Error Annotation}

A comprehensive PEW is finalized by performing QA checks for the project in order to determine whether the desired quality level has been attained, followed by MTevaluation processes and error annotation. Some industry professionals choose to add an additional human revision step to their workflow, which makes the result equal to Translation, Editing and Proofreading (TEP), instead of comparable.

MT evaluation aims at providing quality data for the MT system used, identifying edit patterns and determining whether the engine at hand is appropriate for a specific type of content. This process involves assessing meaning preservation, fluency and PE effort based on some pre-established industry methods, known as automated evaluation models. These models provide highly valuable information to LSPs and MT researchers alike, allowing them to monitor the engine's performance and improve their systems over time, but also constitute a reliable point of reference for linguists themselves, in order to set up appropriate pricing models.

These human-executed processes constitute the final stage of the optimal PE workflow, known as annotation. Annotation consists in the classification and analysis of errors identified by a linguist in the machine translated text in order to not only provide quality data for the specific task and additional information on the MT system's functionality in general but also to keep track of the changes and corrections applied. This usually involves tagging errors according to an industry-standard typology and providing information related to the nature of the edits applied by post-editors depending on the type and frequency of the errors.

There are many ways to collect feedback from the post-editors about any given system. It can be gathered using exhaustive reporting systems and tools ${ }^{1}$. However, feedback may also be collected in a plain Excel file, an email or even a call. What is important is that we allow the post-editors who have worked using the system to give their own opinion about the errors found and use this to improve it (Guerrero, 2018).

\section{The Training Challenges of the PEW}

We may envision the translators at the very center of the post-editing process, using the computer and deciding how to best combine the materials they have on hand at each part of the process, either glossaries, translation memories or machine translation engines (Rico, 2017). The Training Challenges of the PEW, including Pre-Editing, Post-Editing and Error Annotation, are examined in relation to three Groups: Post-Editors, LSPs and Universities based on a digital survey among language industry professionals (Gene, 2020).

\footnotetext{
1 DQF4, which allow for severity and error classification of the output, measurement of MT productivity, ranking of MT engines, evaluation of adequacy and fluency (Sanchez-Martinez, 2018). Recently, a new metric for error typology has been developed based on the harmonization of the MQM and DQF frameworks, and available through an open TAUS DQF API. This harmonization allows errors to be classified, firstly, according to a broader DQF error typology and, subsequently, by the subcategories as defined in MQM.
} 


\subsection{The Training Challenges of the PEW for the LSPs}

For LSPs, it seems that all of the challenges mentioned in Fig. 3 are equally important, which reflects the work that needs to be done and the investment in time, effort, communication, research and training on the LSPs' end.

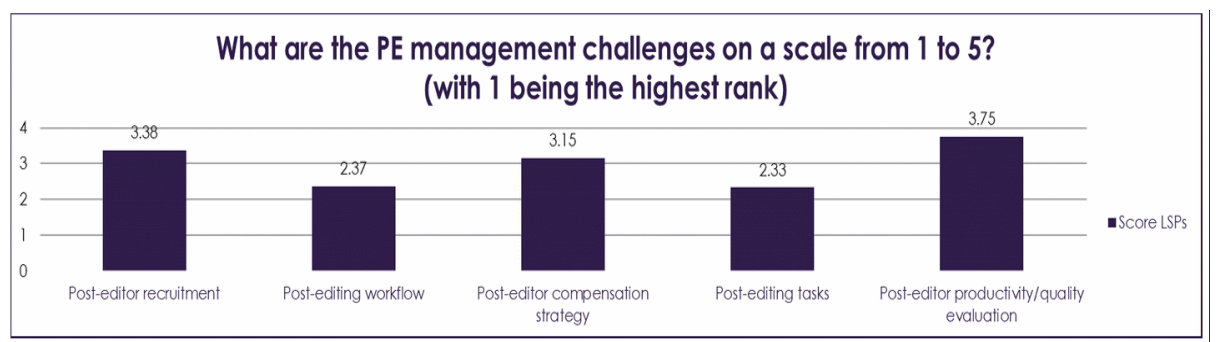

Fig. 1. What are the PE management challenges on a scale from 1 to 5 ? (Gene, 2020)

Training challenges affect all departments of LSPs, from the recruiters and vendor managers who draft the job descriptions and post-editors' profile(s) to the production manager who integrates the post-editing workflow, measures the productivity and applies the post-editor compensation strategy, and the quality manager who is responsible for setting a quality evaluation procedure to measure the quality results.

In a survey conducted as part of the EAMT 2018 21st Annual Conference (PérezMacías, Rico and Forcada, 2018), 52\% of the translators were willing to accept postediting jobs and $79 \%$ considered that translators contribute to MT development:

DEGREE OF CONFORMITY WITH THE FOLLOWING STATEMENTS

\begin{tabular}{r|llllll} 
& $\begin{array}{l}\text { Strongly } \\
\text { agree }\end{array}$ & Agree & Indifferent & Disagree & $\begin{array}{l}\text { Strongly } \\
\text { disagree }\end{array}$ \\
\hline I'mistrust MT & $15 \%$ & $\mathbf{2 9} \%$ & $16 \%$ & $\mathbf{2 9} \%$ & $12 \%$ \\
\hline Translators contribute to MT development & $\mathbf{4 0 \%}$ & $\mathbf{3 9} \%$ & $13 \%$ & $6 \%$ & $2 \%$ \\
MT helps to improve productivity & $\mathbf{2 6 \%}$ & $\mathbf{3 1} \%$ & $17 \%$ & $16 \%$ & $10 \%$
\end{tabular}

Fig. 1. Degree of conformity with several statements about MT

However, it is particularly worrying that, when answering the question 'How often the translators' needs about MT are heard', a total of $40 \%$ answered 'never' or 'almost never'. As part of this question the translators added their suggestions on ways to contribute to the MT process: 




Fig. 1. How often the translators' needs about MT are heard and possible ways to contribute

Based on the above, one of the main challenges for LSPs is mutual collaboration. According to Guerrero (2018) if all parties involved in machine translation processes acknowledge that mutual collaboration is not only possible but also desirable, then the challenge for LSPs and machine translation buyers is to take up the torch from academic research and establish new relationships with post-editors, moving towards a more translator-centered process.

\subsection{The Training Challenges of the PEW for the Post-Editors}

Even if productivity increases while quality is maintained, actual experience shows that PE is a tiring task for translators (Guerberof, 2018), which pushes boundaries and has no clear boundaries as a service.

In the context of Gene's survey (2020), SLVs responses (see Fig. 4) validate the feedback of LSPs (see Fig. 3) prioritizing the lack of training, the lack of post-editing skills with most important the ambiguity of post-editing tasks guidelines, which highlights the importance of mutual collaboration between LSPs and SLVs in terms of training.

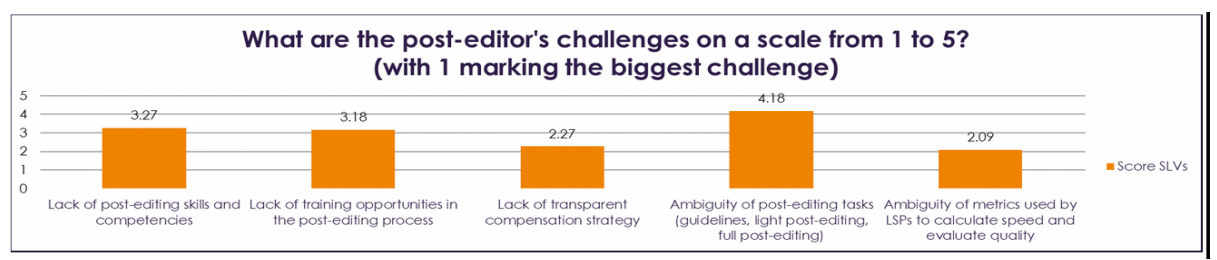

Fig. 1. What are the post-editor's challenges on a scale from 1 to 5? (Gene, 2020)

\subsection{The Training Challenges of the PEW for Academia}

Machine translation and post-editing are little by little finding their place as independent subjects in translation graduate and post-graduate programmes (Guerrero, 2018) ${ }^{2}$.

2 Regarding MT and PE training for translators, the skills needed have been described by O’Brien (2002), Rico and Torrejón (2012) and Pym (2013), while syllabi have been de- 
Based on Gene's survey (2020), LSPs and Universities seem to be isolated with no strong connection link between them. In their majority, SLVs find that the syllabus offered by Universities is not adapted to the translation industry needs, while $30 \%$ of them believe that it does meet the industry needs.

The main challenge for Academia right now is the lack of trainers. As MTPE-related courses have only recently been added to the curricula of some Universities, time will be needed for new students to evolve into future MTPE Trainers who will inspire the translation industry and bridge the training gap between LSPs and PostEditors, balancing the demand and the offer respectively and meeting the quality standards needed.

\section{Conclusions}

As we have attempted to highlight throughout this paper, Post-Editing is actually but one step in the optimal workflow. Industry experience shows that, in practice, Language Service Providers rarely stick to this 3-step comprehensive workflow, with preediting and annotation steps often skipped, and consequently fail to profit from a real return on investment. However, unless pre-editing and annotation procedures are applied we could argue that the whole process is at best ineffective or even futile; without these a Post-Editing project is merely a one-off that simply saves the linguist and client some time through the use of MT technology, but bears no real value for the company supporting it. For this reason, LSPs should revise their standard practices and establish a workflow that is both profitable and sustainable in the long term by making the most of available technologies.

To sum up, on the one hand, LSPs should bear in mind that Post-Editing is best suited to larger volumes (over 100k TUs). On the other hand, they should not forget that human processes such as pre-editing and annotation are the keys to achieving a better return on investment in the long term. Workflows limited to MT and post-editing processes do not constitute an effective strategy with long-term profitable results, but only temporary discounts. In order to enjoy the real benefits of integrating postediting to their list of offered services, an investment should be made in the complete, optimal post-editing workflow.

Regarding the training challenges arising as a result of the PEW, the suggestion is to abandon the current machine-centered paradigm and work with all stakeholders towards a translator-centered process, in which the post-editor is transformed in a translation expert applying critical thinking, problem-solving and decision-making skills not only being involved to correct the errors stubbornly produced by the MT system

signed, and courses explained and described, by Doherty et al. (2012), Doherty and Moorkens (2013), Doherty and Kenny (2014), Koponen (2015) and Mellinger (2017). The training suggestions made by Guerberof (2018) include teaching basic MT technology concepts, MT evaluation techniques, Statistical MT (SMT) training, pre-editing and controlled language, monolingual $\mathrm{PE}$, understanding various levels of $\mathrm{PE}$ (light and full), creating guidelines, MT evaluation, output error identification, when to discard unusable segments and continuous PE practice. 
but having a vital role in each and every step of the workflow (Guerrero, 2018). The derived data on training challenges among the stakeholder will serve for further analysis and speculations for future problem-solving and more specific training pathways. The closer interaction between the Academia and the LSPs during the traineeships with a common training model agreed between them would be interesting to be examined along with the evaluations of LSPs and the queries of Post-Editors, which could feed the PE teaching models of the Universities.

Bridging the distance between the different stakeholders (LSPs, Post-Editors and Academia) through training is the key to the enlargement of the translation industry. Translators should be encouraged to embrace MT processes and companies to integrate post-editors into their processes, investing in the linguist-focused and not the machine-centered processes for higher quality and productivity.

\section{References}

1. Aranberri, N., G. Labaka, A. Diaz de Ilarraza and K. Sarasola (2014), 'Comparison of Post-editing Productivity Between Professional Translators and Lay Users', in S. O'Brien, M. Simard and L. Specia (eds), Proceedings of the 3rd workshop on post-editing technology and practice, 20-33, Vancouver: AMTA.

2. Arthern, P. J. (1979), 'Machine Translation and Computerized Terminology Aystems: A Translator's Viewpoint', B. M. Snell (ed.), Translating and the Computer: Proceedings of a seminar, 77-108, London: North-Holland.

3. Aziz, W., S. Castilho and L. Specia (2012), 'PET: A Tool for Post-editing and Assessing Machine Translation', in Proceedings of the Eight International Conference on Language Resources and Evaluation (LREC'12), 3982-3987, Istanbul: European Language Resources Association (ELRA).

4. Bartolomé Mesa-Lao, bm.ibc@cbs.dk, Introduction to post-editing, Center for Research and Innovation in Translation and Translation Technology, Copenhagen Business School, Denmark

5. Zainurrahman Sehan, STKIP Kie Raha Ternate, Five Translation Competencies

6. Celia Rico, Senior Lecturer, Facultad de Artes y Comunicación, Universidad Europea de Madridcelia.rico@uem.es, Enrique Torrejón, Language Technologies Consultantenrique.torrejon@gmail.com, Skills and Profile of the New Role of the Translator as MT Posteditor

7. Allen, J. (2003). Post-Editing. In H. Somers (Ed.), Computers and Translation: a Translator's Guide (pp. 297-317). Amsterdam: John Benjamins.

8. Aranberri, N. (2017). What Do Professional Translators Do When Post-Editing for the First Time? First Insight Into the Spanish-Basque Lanuage Pair. HERMES - Journal of Language and Communication in Business(56), 89-110.

9. Bentivogli, L., Bisazza, A., Cettolo, M., \& Federico, M. (2016). Neural Versus PhraseBased Machine Translation Quality: a Case Study. Conference on Empirical Methods in Natural Language Processing, (pp. 257-267). Austin, Texas.

10. Blagodarna, O. (2018). 'Enhancement of Post-Editing Performance: Introducing Machine Translation Post-Editing in Translator Training', Universidad Autonoma de Barcelona.

11. Blanchon, H., \& Besacier, L. (2017, September 21). Comparing Statistical Machine Translation (SMT) and Neural Machine Translation (NMT) Performance. Retrieved from http://lig-membres.imag.fr/blanchon/SitesEns/NLSP/resources/SMT-vs-NMT.pdf 
12. Daems, J., Vandepitte, S., Hartsuiker, R. J., \& Macken, L. (2017). Identifying the Machine Translation Error Types with the Greatest Impact on Post-editing Effort. Frontiers in Psychology, 8, 1282. doi:https://doi.org/10.3389/fpsyg.2017.01282

13. de Almeida, G. (2013). Translating the post-editor: an investigation of post-editing changes and correlations with professional experience across two Romance languages. Dublin: Dublin City University.

14. Dino, G. (2017, December 18). 3 Reasons Why Neural Machine Translation is a Breakthrough. Retrieved from Slator: Language Industry Intelligence:

15. Gene, V. (2020, May 21). The Management and Training Challenges of Post-Editing (Part 2). Retrieved from GALA: Globalization and Localization Association: https://www.galaglobal.org/ondemand/management-and-training-challenges-post-editing-part-2

16. Gene, V. (2020, May 7). The Management and Training Challenges of Post-Editing (Part 1). Retrieved from GALA: Globalization and Localization Association: https://www.galaglobal.org/ondemand/management-and-training-challenges-post-editing-part-2

17. Gene, V. (2020, November). The Role and Perspective of the Post-Editor: What Are the Training Challenges?, Translating and the Computer - TC42. London.

18. Ginovart Cid, C., Colominas, C., \& Oliver, A. (2020). Language Industry Views on the Profile of the Post-editor. Translation Spaces. doi:https://doi.org/10.1075/ts.19010.cid

19. Guerberof Arenas, A., \& Moorkens, J. (2019, January). Machine Translation and Postediting Training as Part of a Master's Programme. The Journal of Specialised Transla$\operatorname{tion}(31), 217-238$.

20. Guerrero, L. (2018). From a Discreet Role to a Co-Star: The Post-Editor Profile Becomes Key to the Post-Editing Workflow. Translating and the Computer - TC40. London.

21. Hu, K., \& Cadwell, P. (2016). A Comparative Study of Post-Editing Guidelines. Baltic Journal of Modern Computing, 4(2), 346-353.

22. Koponen, M. (2015), 'How to Teach Machine Translation Post-editing? Experiences from a Post-editing Course', in S. O'Brien, M. Simard and L. Specia (eds), Proceedings of the 4th Workshop on Post-editing Technology and Practice (WPTP 4), 2-15, Miami: AMTA.

23. Koponen, M., W. Aziz, L. Ramos and L. Specia (2012), 'Post-editing Time as a Measure of Cognitive Effort', in S. O'Brien, M. Simard and L. Specia (eds), Proceedings AMTA 2012 Workshop on Post-editing Technology and Practice, 11-20, San Diego: AMTA.

24. Krings, H. P. (2001). Repairing texts: Empirical investigations of machine translation postediting processes. Kent, Ohio: Kent State University Press.

25. Lommel, A. R. (2017), 'Neural MT: Sorting Fact from Fiction', Common Sense Advisory. Available online: http://www.commonsenseadvisory.com/AbstractView/tabid/74/ArticleID/37893/Title/NeuralMTSortingFactfromFiction/Default.aspx (accessed 21 September 2018).

26. Lommel, A. R. and D. A. De Palma (2016a), 'Post-editing Goes Mainstream', Common Sense Advisory. Available online: (accessed 21 September 2018).

27. Lommel, A. R. and D. A. De Palma (2016b), 'TechStack: Machine Translation', Common Sense Advisory. Available online: (accessed 21 September 2018).

28. Mellinger, C. D. (2017), 'Translators and Machine translation: Knowledge and Skills Gaps in Translator Pedagogy', The Interpreter and Translator Trainer, 1-14, Taylor \& Francis Online.

29. Moorkens, J. and S. O'Brien (2013), 'User Attitudes to the Post-editing Interface', in S. O'Brien, M. Simard and L. Specia (eds), Proceedings of 2nd Workshop on Post-editing Technology and Practice, 19-25, Nice: EAMT.

30. Moorkens, J. and S. O'Brien (2015), 'Post-editing Evaluations: Trade-offs Between Novice and Professional Participants', in Proceedings of EAMT, 75-81, Antalya: EAMT. 
31. Nitzke, Jean. 2019. Problem solving activities in post-editing and translation from scratch: A multi-method study (Translation and Multilingual Natural Language Processing 12). Berlin: Language Science Press.

32. Nunes Vieira, L., Alonso, E., \& Bywood, L. (2019). Introduction: Post-editing in practice - Process, product and networks. The Journal of Specialised Translation, 2-13.

33. O’Brien, S., (2002). Teaching Post-editing: A Proposal for Course Content, 6th EAMT Workshop Teaching Machine Translation.

34. O'Brien, S., Roturier, J., \& de Almeida, G. (2009). Post-Editing MT Output; Views from the researcher, trainer, publisher and practitioner. Retrieved from http://www.mt-archive.info/MTS-2009-OBrien-ppt.pdf

35. O'Brien, S., \& Moorkens, J. (2014). Towards Intelligent Post-Editing Interfaces. In W. Baur, B. Eichner, S. Kalina, N. Kessler, F. Mayer, \& J. Orsted (Ed.), 20th FIT World Congress, (pp. 131-137). Berlin.

36. O'Brien, S., Winther Balling, L., Carl, M., Simard, M., \& Specia, L. (Eds.). (2014). Postediting of Machine Translation: Processes and Applications. Newcastle: Cambridge Scholars Publishing

37. O'Brien, S. and S. Castilho (2016), 'Evaluating the Impact of Light Post-editing on Usability', in Proceedings of the Tenth International Conference on Language Resources and Evaluation (LREC'16), 310-6, Portorož: European Language Resources Association (ELRA).

38. O'Brien, S. (2017), 'Machine Translation and Cognition', The Handbook of Translation and Cognition, J. W. Schwieter and A. Ferreira (eds), 311-31, Wiley-Blackwell.

39. Pym, A. (2013), 'Translation Skill-sets in a Machine-translation Age', Meta, 58 (3), 487503.

40. Rico, C., \& Torrejon, E. (2012). Skills and Profile of the New Role of the Translator as MT Post-Editor. Tradumàtica: tecnologies de la traducció, 166-178.

41. Saillard, J.-L. (2019, January 28). Transitioning to a post-editing machine translation business model. Retrieved from Smartcat: https://www.smartcat.com/blog/transitioning-to-apost-editing-machine-translation-business-model/

42. Sanchez-Martinez, F. (2012). "Motivos del creciente uso de la traduccion automatica seguida de posedicion". Tradumatica, 10, 150-156 [Download on 8 March 2015].

43. TAUS. (2011a). Adequacy/Fluency Evaluation [Download on 4 March 2015].

44. TAUS. (2011b). Adequacy/Fluency Process Guide [Download on 4 March 2015].

45. TAUS. (2011c). Dynamic Quality Framework [Download on 4 March 2015].

46. TAUS. (2011d). Error Typology [Download on 4 March 2015].

47. TAUS. (2014, January 27). Evaluating Post-Editor Performance Guidelines. Retrieved from TAUS: the language data network: https:/www.taus.net/academy/best-practices/ postedit-best-practices/evaluating-post-editor-performance-guidelines

48. Tyler, S., Hertel, P., \& McCallum, M. H. (1979). Cognitive Effort and Memory. Journal of Experimental Psychology: Human Learning and Memory, 5(6), 607-617.

49. Vashee, K., \& Wiggins, D. (2014). A 6 Step Plan to Create an Optimal MT Post-Editing Strategy. Retrieved from https://omniscien.com/wp-content/uploads/2016/02-new/AsiaOnline-Webinar-A-6-Step-Plan-to-Create-an-Optimal-MT-Post-Editing-Strategy2.pdf

50. Vasconcellos, M. and M. León (1985): "SPANAM and ENGSPAN: Machines Translation at the Pan American Health Organization", Computational Linguistics 11, 122-136. [online] Available. http://acl.ldc.upenn.edu/J/J85/J85-2003.pdf [accessed 13 September 2012]

51. Zainurrahman, S. (2010). Five Translation Competencies. Retrieved from Zainurrahman's Personal Journal: https://zainurrahmans.wordpress.com/2010/06/06/five-translation-competencies/ 
52. Zaretskaya, A. (2019). Optimising the Machine Translation Post-editing Workflow. In I. Temnikova, C. Orasan, G. Corpas Pastor, \& R. Mitkov (Ed.), 2nd Workshop on Human-Informed Translation and Interpreting Technology, (pp. 136-139). Varna.

53. Zaretskaya, A., Vela, M., Corpas Pastor, G., \& Seghiri, M. (2016). Measuring Post-editing Time and Effort for Different Types of Machine Translation Errors. New Voices in Translation Studies, 15, 63-91.

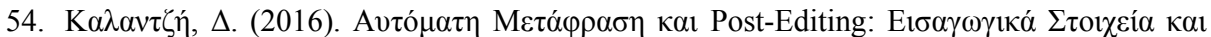
$\Sigma \kappa \varepsilon ́ \psi \varepsilon ı \varsigma$. th Meeting of Greek Translatologists. 